\title{
ANATOMICAL ARRANGEMENT OF THE SUBCLAVIAN ARTERY BRANCHES IN THE RABBIT AND EUROPEAN HARE
}

\author{
Maženský, D., Flešárová, S. \\ Department of Anatomy, Histology and Physiology \\ University of Veterinary Medicine and Pharmacy, Komenského 73, 04181 Košice \\ Slovakia \\ david.mazensky@uvlf.sk
}

\section{ABSTRACT}

The aim of this study was to compare the anatomical arrangements of the branches arising from the subclavian arteries in the domesticated rabbit and hare. The study was carried out on ten adult rabbits and ten adult European hares using the corrosion cast technique. After the euthanasia, the vascular network was perfused with saline. The arterial system of the entire body was injected by Batson's corrosion casting kit No.17. After polymerization of the medium, the maceration was carried out in $\mathrm{KOH}$ solution. The arrangement of the origins of the branches of the bilateral subclavian arteries were more variable in the hare. The number of branches arising from the subclavian artery were more regular in the rabbit on the right side and in the hare on the left side. In the rabbit, we found in two cases, the origins of the branches of the left subclavian artery from the aortic arch. The anatomical differences found between the rabbit and the hare may possibly be associated with their different ways of life.

Key words: European hare; origin; rabbit; subclavian artery

\section{INTRODUCTION}

The subclavian arteries of both sides of the body continue cranially to reach the cranial border of the first rib forming a convex curve. After their turn, they continue into the corresponding thoracic limb as the axillary arteries.

The branches arising from the subclavian artery are the vertebral artery, deep cervical artery, dorsal scapular artery and supreme intercostal artery. Some or all of these branches can be fused to form a common stem of origin known as the costocervical trunk. The subclavian artery gives off at the level of thoracic entrance the internal thoracic artery and superficial cervical artery $[6,8]$.

The vertebral artery emerges from the thoracic cavity through the thoracic entrance. It then passes through the transverse foramen of the sixth cervical vertebra into the transverse canal of the cervical vertebrae, in which it continues in the cranial direction. It supplies the cervical part of the spinal cord, brain, meninges, cervical vertebral bodies and neighboring musculature.

The deep cervical artery leaves the thoracic cavity through the first intercostal space and supplies the muscles of the neck from the withers to the nape. The dorsal scapular artery after leaving the thoracic cavity through 
the first intercostal space supplies the muscles and skin of the withers $[6,8]$. The supreme intercostal artery continues caudally on the ventral surface of the heads of the ribs and gives off the dorsal intercostal arteries supplying the first four intercostal spaces $[6,7]$. The internal thoracic artery is directed ventrocaudally on the dorsal surface of the sternum. It participates by supplying blood to the ventral part of lateral thoracic wall, sternum, thoracic mammary complexes, mediastinum, pericardium, thymus, diaphragm, abdominal musculature and skin. The superficial cervical artery is directed to the lateral surface of the external jugular vein. This artery supplies the cervical lymph-nodes and neighboring musculature $[6,8]$.

The literature dealing with the arterial system in the rabbit and hare in detail is rather [6, 7]. The aim of this study was to describe the origin, localization and variations of the subclavian artery branches in two related species: the domesticated rabbit and European hare.

\section{MATERIALS AND METHODS}

This study was carried out on 9 adult European hares (Lepus Europaeus, L. 1758, age 140 days) and on 9 adult domesticated rabbits (Oryctolagus cuniculus $f$. domestica, L. 1758, of the same age). We used hares (obtained from ISFA APRC, Nitra, Slovakia) of both sexes (female $n=5$; male $\mathrm{n}=5$ ), with a weight range between $2.5-3.2 \mathrm{~kg}$ and New Zealand White rabbits (obtained from HYLAPA s.r.o., Prešov, Slovakia) of both sexes (female $n=5$; male $n=5$ ), in an accredited experimental laboratory of the University of Veterinary Medicine and Pharmacy in Košice, Slovakia. The animals were kept in cages under standard conditions (temperature $15-20^{\circ} \mathrm{C}$, relative humidity $45 \%$, 12-hour light period), and fed with a granular feed mixture (O-10 NORM TYP, Spišské kŕmne zmesi, Spišské Vlachy, Slovakia). The drinking water was available to all animals ad libitum. The animals were injected intravenously with heparin (50 000 IU. $\mathrm{kg}^{-1}$ ) $30 \mathrm{~min}$ before they were sacrificed by intravenous injection of embutramide (T-61, $\left.0.3 \mathrm{ml} . \mathrm{kg}^{-1}\right)$. Immediately after euthanasia, the vascular network was perfused with a physiological solution. During manual injection through the ascending aorta, the right atrium of the heart was opened in order to lower the pressure in the vessels which enabled an optimal injection distribution. $50 \mathrm{ml}$ of Batson's corrosion casting kit No. 17 (Dione, České Budějovice, Czechia) was used as the casting medium. The maceration was carried out in $2-4 \% \mathrm{KOH}$ solution for a period of 5 days at $60-$ $70^{\circ} \mathrm{C}$. The study was carried out under the authority decision No.2647/07-221/5.

\section{RESULTS}

\section{Rabbit}

In all specimens, the left subclavian artery arose from the aortic arch as the second branch after the origin of the brachiocephalic trunk. The left subclavian artery gave off, two branches in one case, three branches in two cases, four branches in two cases and five branches in four cases. In one case, a common trunk for the left supreme intercostal artery, left deep cervical artery, left dorsal scapular artery and left internal thoracic artery arose directly from the aortic arch caudally to the origin of the left subclavian artery. Also in one case, the left vertebral artery arose from the aortic arch as an independent branch cranially to the origin of the left subclavian artery.

In two cases, the first branch originating from the left subclavian artery was the left supreme intercostal artery (Fig. 1) and also in two cases a common trunk dividing into the left supreme intercostal artery, left deep cervical artery and left dorsal scapular artery. In one case, the first branch was the left vertebral artery, left superficial cervical artery, left internal thoracic artery, a common trunk for the left supreme intercostal artery, left deep cervical artery, left dorsal scapular artery and left internal thoracic artery, and a common trunk for the left supreme intercostal artery and left deep cervical artery. A common trunk consisting of the left deep cervical artery and left dorsal scapular artery was present as the second branch in three cases (Fig. 1). The left vertebral artery as the second branch was present also in three cases. In two cases, the second branch was the left internal thoracic artery and in one case the left superficial cervical artery. The third branch was formed as follows: the left vertebral artery in three cases (Fig. 1), the left internal thoracic artery in two cases, the left superficial cervical artery in two cases and the left supreme intercostal artery in one case. In two cases, the left superficial cervical artery was present as the fourth branch. In one case, were present as the fourth branch of the left vertebral artery, the left superficial cervical artery, a common trunk for the left deep cervical artery and left dorsal scapular artery, and a com- 


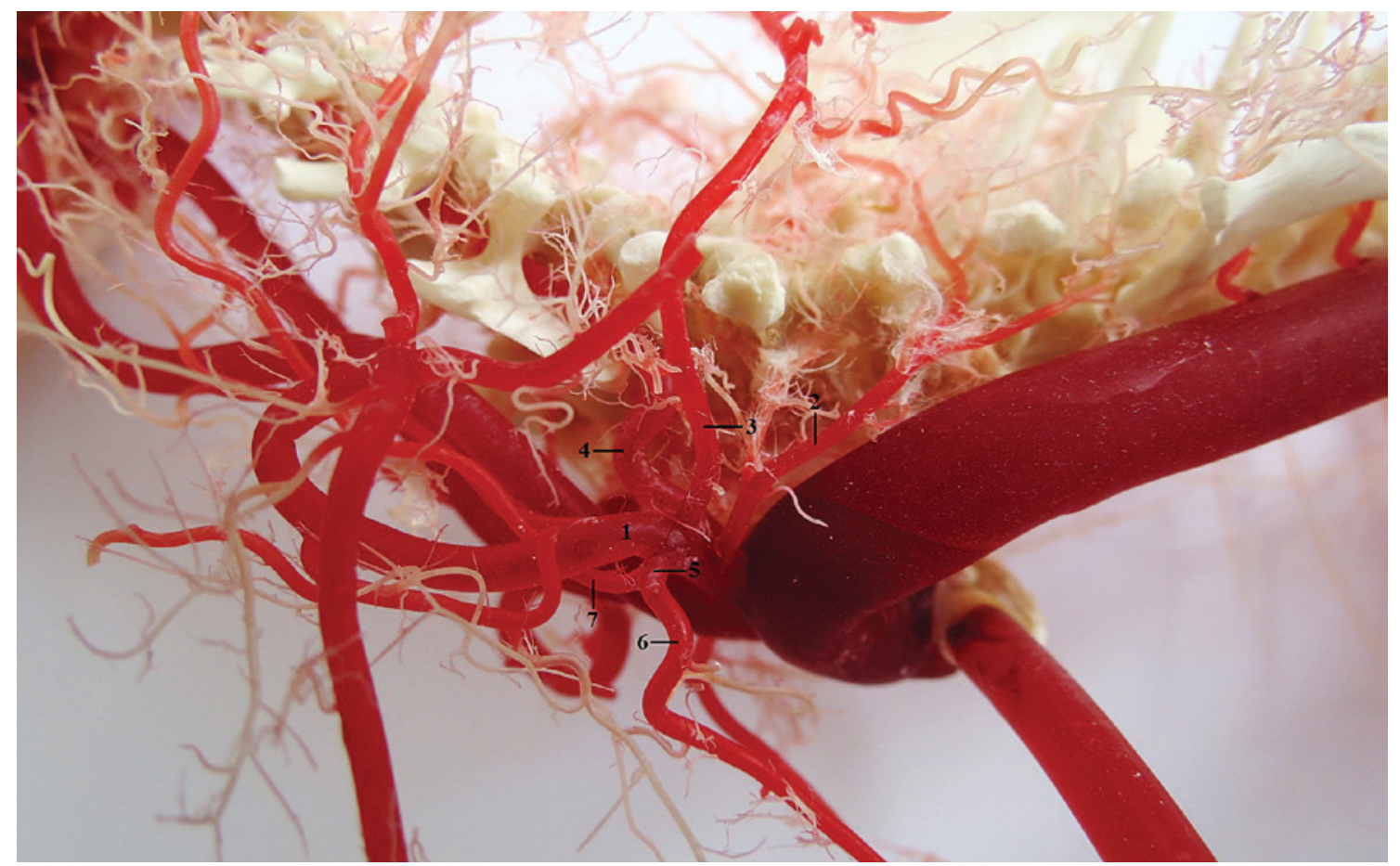

Fig. 1. The branches of the left subclavian artery in the rabbit

1 - left subclavian artery; 2 - left supreme intercostal artery; 3 - common trunk for the left dorsal scapular artery and left deep cervical artery; 4 - left vertebral artery; 5-common trunk; 6-left internal thoracic artery; 7 - left superficial cervical artery. Macroscopic image, dorsolateral view

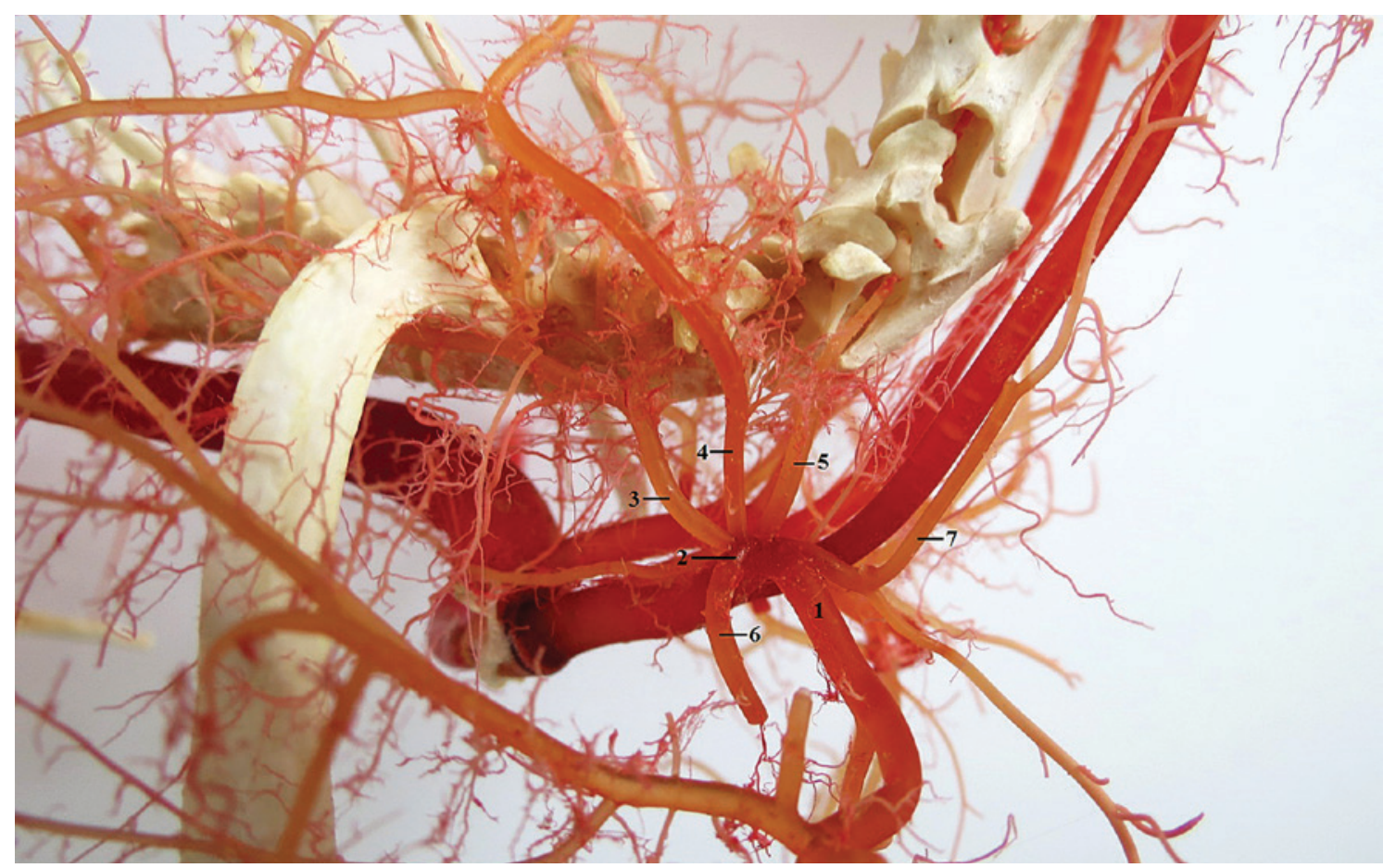

Fig. 2. The branches of the right subclavian artery in the hare

1 - right subclavian artery; 2 - common trunk; 3 - right supreme intercostal artery; 4 - common trunk for the right dorsal scapular artery and right deep cervical artery; 5 - right vertebral artery; 6 - right internal thoracic artery; 7 - right superficial cervical artery. Macroscopic image, craniolateral view 
mon trunk formed by the left internal thoracic artery and left superficial cervical artery (Fig. 1). The left superficial cervical artery or the left internal thoracic artery constituted the fifth branch in two cases.

The right subclavian artery originated from the brachiocephalic trunk in all specimens. Three branches of the right subclavian artery were present in five cases and four branches in four cases.

As the first branch in three cases was present a common trunk for the right supreme intercostal artery, right deep cervical artery, right dorsal scapular artery and right internal thoracic artery or the right vertebral artery. In two cases, the first branch was represented by the right superficial cervical artery and in one case by the first right superficial cervical artery. The second branch showed the following arrangement: the right vertebral artery in four cases, the right internal thoracic artery in three cases and a common trunk for the right supreme intercostal artery, right deep cervical artery, right dorsal scapular artery and right internal thoracic artery in one case. The right superficial cervical artery as the third branch originated in four cases. In two cases, the right vertebral artery represented the third branch. In one case, as the third branch was present, the doubled right superficial cervical artery, a common trunk for the right supreme intercostal artery, right deep cervical artery, right dorsal scapular artery and right internal thoracic artery or a common trunk for the right supreme intercostal artery, right deep cervical artery and right dorsal scapular artery. A common trunk for the right supreme intercostal artery, right deep cervical artery and right dorsal scapular artery was present as a fourth branch in two cases. In one case, it was the right superficial cervical artery and the second was the right superficial cervical artery.

\section{European hare}

The left subclavian artery originated from the aortic arch in all corrosion casts. It gave off five branches in eight cases and four branches in one case.

The left supreme intercostal artery was the first branch in all cases. The left internal thoracic artery was the second branch in seven cases, and a common trunk for the left deep cervical artery and left dorsal scapular artery in two cases. The third branch was, in six cases a common trunk for the left deep cervical artery and left dorsal scapular artery; in one case the left vertebral artery; in one case the left internal thoracic artery; and also in one case a com- mon trunk for the left vertebral artery, left deep cervical artery and left dorsal scapular artery. The left vertebral artery was found as the fourth branch in seven cases and the left superficial cervical artery in two cases. The left superficial cervical artery represented the fifth branch in seven cases and the left internal thoracic artery in one case.

The right subclavian artery as a branch of the brachiocephalic trunk was present in all specimens, and it gave off three branches in five cases, five branches in three cases and six branches in one case.

The first branch showed the following arrangement: a common trunk for the right supreme intercostal artery, right deep cervical artery, right dorsal scapular artery and right internal thoracic artery in five cases (Fig. 2), the right internal thoracic artery in two cases, the right supreme intercostal artery in one case and the right superficial cervical artery also in one case. The second branch was formed in six cases by the right vertebral artery (Fig. 2), in two cases by the right supreme intercostal artery and in one case by the right internal thoracic artery. The right superficial cervical artery was found as the third branch in five cases (Fig. 2), a common trunk for the right deep cervical artery and right dorsal scapular artery in three cases and the right deep cervical artery in one case. The right vertebral artery represented the fourth branch in two cases, the right supreme intercostal artery in one case, and the right dorsal scapular artery also in one case. The right superficial cervical artery was the fifth branch in two cases, the right internal thoracic artery in one case and the right vertebral artery also in one case. In one case, was formed the sixth branch by the right superficial cervical artery.

\section{DISCUSSION}

The origin, course and direction of the subclavian arteries in our corrosion casts of the rabbit arterial system were similar to the observations of Craigie [1] and Popesko et al. [7]. From the left subclavian artery originated three branches [7] or five branches [1], but in our specimens their number varied from two to five.

As the first branch arising from the left subclavian artery we found a common trunk for the left supreme intercostal artery, the left deep cervical artery and the left dorsal scapular artery [7]. The same arrangement was found only in two cases. Also, in two cases, the first branch was the left 
supreme intercostal artery. The left vertebral artery, left superficial cervical artery, left internal thoracic artery, a common trunk for the left supreme intercostal artery, left deep cervical artery, left dorsal scapular artery and left internal thoracic artery, and a common trunk for the left supreme intercostal artery and left deep cervical artery were the first branch only in one case. The second branch in our specimens was a common trunk for the left deep cervical artery and left dorsal scapular artery in three cases, the left vertebral artery in two cases, the left internal thoracic artery in two cases and the left superficial cervical artery in one case. Popesko et al. [7] described the left internal thoracic artery as the second branch. The left vertebral artery is the third branch arising independently from the left subclavian artery (Popesko et al. [7]). In our specimens, the third branch was represented by: the left vertebral artery in three cases, the left internal thoracic artery in two cases, the left superficial cervical artery in two cases and the left supreme intercostal artery in one case.

The origin, course and direction of the right subclavian artery in our specimens have been covered previously by the descriptions of Craigie [1] and Popesko et al. [7]. The right subclavian artery gives off five [1] or three branches [7]. In our specimens, three branches were present in five cases and four branches in four cases.

The first branch found by Popesko et al. [7] was a common trunk for the right supreme intercostal artery, right deep cervical artery, right dorsal scapular artery and right vertebral artery. The same arrangement of the first branch was present in our specimens in three cases. In two cases, the right superficial cervical artery and in one case the first right superficial cervical artery represented the first branch. The second branch showed the following arrangement: the right vertebral artery in four cases, the right internal thoracic artery in three cases and a common trunk for the right supreme intercostal artery, right deep cervical artery, right dorsal scapular artery and right internal thoracic artery in one case. Popesko et al. [7] described the right internal thoracic artery as the second branch. In the literature, the third branch was designated as the right superficial cervical artery (Popesko et al. [7]). The same arrangement was present in our specimens in four cases. In the rest of the cases, the third branch was formed as follows: the doubled right superficial cervical artery in one case, a common trunk for the right supreme intercostal artery, right deep cervical artery, right dorsal scapular ar- tery and right internal thoracic artery in one case, common trunk for the right supreme intercostal artery, right deep cervical artery and right dorsal scapular artery in one case.

The vertebral artery, superficial cervical artery, deep cervical artery, supreme intercostal artery and the internal thoracic artery as subclavian artery branches without order of their origins were listed by Craigi e [1].

The arrangement of origins of the branches of the bilateral subclavian arteries were more variable in the hare. The number of branches arising from the subclavian artery was more regular in the rabbit on the right side and in the hare on the left side. In the rabbit, we found in two of the cases, the origins of the branches of the left subclavian artery from the aortic arch.

The detailed knowledge of the arrangement of the arterial system still represents an extensive gap in the literature. We believe that the anatomical knowledge contributes to a better understanding of behavioral differences between familiar species in domesticated as well as in experimental studies and the veterinary daily practice, too $[2,3,4,5]$.

\section{CONCLUSIONS}

The results of our study indicated high variability in the arrangement of the branches arising from the bilateral subclavian arteries. The anatomical differences found between the rabbit and the hare are possibly associated with the different ways of life.

\section{REFERENCES}

1. Craigie, E. H., 1948: Bensley's Practical Anatomy of the Rabbit. Blakiston Company, Philadelphia, 325-326.

2. Dugat, D., Rochat, M., Ritchey, J., Payton, M., 2011: Quantitative analysis of the intramedullary arterial supply of the feline tibia. Vet. Comp. Orthop. Traumatol., 24, 313-319.

3. Hossmann, K. A., 1998: Experimental models for the investigation of brain ischemia. Cardiovasc. Res., 39, 106-120.

4. Iwama, H., Akama, Y., Tase, C., 2000: Global brain ischemia produced by clamping left subclavian artery and bicarotid trunk in the rabbit. Am. J. Emerg. Med., 18, 31-35.

5. Mazensky, D., Danko, J., 2010: The importance of the origin of vertebral arteries in cerebral ischemia in rabbit. Anat. Sci. Int., 85, 102-104. 
6. Nickel, R., Schummer, A., Seiferle, E., 1981: The Anatomy of the Domestic Mammals. The Circulatory System, the Skin, and the Cutaneous Organs of the Domestic Mammals. Verlag Paul Parey, Berlin, 72-77.

7. Popesko, P., Rajtova, V., Horak, J., 1990: Anatomic Atlas of Small Laboratory Animals I. 1st edn., Príroda, Bratislava, $67-78$.
8. Popesko, P., Hajovska, B., Marvan, F., Komarek, V., Vrzgulova, M., 1992: Anatomy of Farm Animals. Príroda, Bratislava, 396-399.

Received September 1, 2017

Accepted September 25, 2017 